Cahiers de recherches médiévales

\title{
L'injustice de la justice dans les contes de Bonaventure des Périers
}

Jean-Claude Arnould

\section{CpenEdition}

\section{Journals}

Édition électronique

URL : https://journals.openedition.org/crm/11710

DOI : $10.4000 / \mathrm{crm} .11710$

ISSN : 1955-2424

Éditeur

Honoré Champion

Édition imprimée

Date de publication : 20 novembre 2009

Pagination : 267-278

ISSN : $1272-9752$

Référence électronique

Jean-Claude Arnould, "L'injustice de la justice dans les contes de Bonaventure des Périers ", Cahiers de recherches médiévales [En ligne], 18 | 2009, mis en ligne le 15 décembre 2012, consulté le 15 décembre 2022. URL : http://journals.openedition.org/crm/11710; DOI : https://doi.org/10.4000/crm. 11710 


\title{
酶M
}

\author{
L'injustice de la justice \\ dans les contes de Bonaventure des Périers
}

\begin{abstract}
The Nouvelles Récréations et Joyeux Devis (1558) of Bonaventure des Périers present many confrontations between lawyers and outlaws. The satirical aspect of these tales has been acknowledged by critics, but the analysis of these texts reveals their more radical meaning and the way they question the reality of the justice. Emphasizing the unfairness of judges and law courts, Des Périers claims the right to a natural and universal justice contrasting with so deeply perverted human laws.

Résumé: Les Nouvelles Récréations et Joyeux Devis (1558) de Bonaventure des Périers présentent de multiples confrontations entre gens de justice et hors-la-loi. Si la portée satirique en a été reconnue par la critique, l'analyse de ces récits révèle la signification plus radicale qu'ils prennent, en mettant en cause la réalité de la justice. Dévoilant les divers aspects de l'injustice des institutions et des hommes qui en ont la charge, Des Périers affirme contre ces lois humaines profondément viciées les droits d'une justice naturelle et universelle.
\end{abstract}

De «nouvelles récréations », de « joyeux devis » peuvent-ils avoir part à une réflexion sur la loi et la justice?

Le recueil narratif de Bonaventure des Périers s'inscrit dans une parenthèse temporelle : moment de réjouissance que l'on hâte dans l'attente d'une paix longue à venir $\left.{ }^{1}\right)$, mais aussi temps de plaisir que l'on s'octroie avant qu'adviennent des « siecles malheureux $»^{2}$. L'art de «tromper le temps $»^{3}$ allègera les contraintes qui pèsent sur l'esprit comme, dans le sonnet initial, «chagrin », « ire» et « discours », « tristes passions » et « occupations » cèdent quelque espace à « folie » et «plaisir»: la facétie ménage une suspension des tourments quotidiens, des délais ${ }^{4}$ et des débats 5 de la vie ordinaire.

\footnotetext{
${ }^{1}$ B. Des Périers, Nouvelles Récréations et Joyeux Devis, éd. K. Kasprzyk (notre édition de référence), Paris, STFM, 1980, p. 13.

${ }^{2}$ Ibid., p. 313.

${ }^{3}$ Ibid., p. 13.

${ }^{4}$ Ibid., p. 14 : «Une trop grande patience vous consume : Ung taire vous tient gehenné...»

${ }^{5}$ Ibid., p. 15 : «...comme les ans ne sont que pour payer les rentes, aussi les noms ne sont que pour faire debatre les hommes. ». Ce refus d'argumenter se traduit par les constantes échappatoires à la discussion, ibid., p. 15 : «Mais laissons là ces beaux enseignemens »; ibid., p. 15 : «il n’y a point de sens allegoricque, ...»; ibid., p. $15:$ : Et ne me venez point demander... Qu'on ne me vienne non plus faire des difficultés», « Ne vous souciez point si...»; ibid., p. 16 : «Que me chault il... ? »; ibid., p. 17 : «Ha ha c'est trop argué »; ibid., XLV, p. 186: «Je devrois payer l'amende pour m'apprendre à philosopher ». Le conteur pratique aussi à titre ironique, la suspension du jugement (ibid., LXXIX, p. 282 et XC, p. 312).
}

Cahiers de Recherches Médiévales, 18, 2009 
Si cette philosophie primitive de la joie éloigne un temps les « fascheries », la facétie permet aussi d'alléger la pression des normes, celles du langage, que taquine un narrateur volubile, celles de la société, auxquelles ses personnages jouent mille tours. Loin de l'exemplarité appliquée des Comptes du Monde adventureux, ou de celle, pressante et oppressante, des histoires tragiques, la posture du conteur pourrait n'être que celle d'un joueur. Et pourtant, les récits mettant aux prises gens de loi et hors-la-loi font entendre une voix sérieuse qui s'interroge, par le divertissement et parfois sur un mode plus grave, sur la transgression et son châtiment et qui met en question la réalité même de la justice.

L'intervention dans les Nouvelles Récréations et Joyeux Devis d'acteurs appartenant au monde du droit relève pour une part de la pure satire.

L'aventure du « docteur en la faculté de Décret » renversé par un bœuf en est un bon exemple: son état est claironné dès l'attaque du conte ${ }^{6}$ et quelques indices viennent enrichir le portrait ${ }^{7}$. Mais l'original du Pogge ne mentionnait pas la qualité de l'acteur $^{8}$ et la portée satirique est donc du fait du conteur français. Ce récit pourrait aussi bien représenter, avec des effets similaires, un autre «malade d'aprehension » (XI, p. 63) - un abbé ou un gentilhomme, par exemple - et il ne met nullement en cause le droit canon. Comme le simple barbier qui oppose son bon sens à la folie de ce docteur, le palefrenier qui revend à un «conseillier du palais » sa propre mule (XXV) illustre la revanche des humbles que peut signifier la facétie? La nouvelle suivante montre d'ailleurs comment, suivant le même principe compensatoire, «...les gaudisseries retournent quelque foys sus leurs gaudisseurs » (p. 125); elle ne concerne pas des gens de justice.

Ces contes correspondent à une orientation bien repérée du recueil, la satire des demi-lettrés ${ }^{10}$, et obéissent à une mécanique fondamentale de la facétie, le renversement de situation, narration d'un bouleversement factuel qui opère une

${ }^{6} \mathrm{Ibid}$., XI, p. 61 : «Un docteur en la faculté de Decret, passant pour aller lire aux escolles...» ${ }^{7}$ Il n'a pas quitté Paris depuis quarante ans, l'accident suscite un grand émoi dans la faculté car il allait y «lire»; L. Sozzi, voit même qu'il utilise le prétexte de l'accident pour faire «sauter» un cours («La satire du monde intellectuel dans les contes de Des Périers», Regards sur la Renaissance 1, 1993, éd. du Cygne, Amboise, p. 62).

${ }^{8} \mathrm{La} 129^{\mathrm{e}}$ facétie présente l'acteur comme «contribulis » du narrateur; Des Périers ajoute d'ailleurs tout le cadre « réaliste » de l'histoire.

${ }^{9}$ Le conte précise que le palefrenier avait été serviteur du conseiller, et qu'il soignait cette mule. Cette fonction de « revanche » de la facétie est soulignée par H. Weber : «La facétie et le bon mot du Pogge à Des Périers ", Humanism in France at the end of the Middle Ages and in the early Renaissance, éd. AHT Levi, Manchester-New York, Barnes \& Nobles, 1970, p. 101 ; v. aussi L. Sozzi, «Tendances politiques et sociales chez les conteurs du XVI siècle », Culture et politique en France à l'époque de l'humanisme et de la Renaissance, éd. F. Simone, Turin, Académie des Sciences, 1974, p. 256-257.

${ }^{10}$ L. Sozzi, Les Contes de Bonaventure Des Périers. Contribution à l'étude de la nouvelle française de la Renaissance, Turin, G. Giappichelli, 1964, p. 382-389. 
remise en ordre salutaire ${ }^{11}$. Si l'on peut dire qu'ils confortent ou rétablissent un ordre de valeurs, ils ne mettent pas en jeu le droit ni la justice à proprement parler, mais vont simplement de pair avec d'autres traits décochés contre l'ensemble des professions qui peuvent alors servir de cible ${ }^{12}$ : dans l'histoire d'un prêtre glouton, les avocats qui «mangent» leurs clients s'inscrivent dans une liste de possibles comprenant marchand, boucher et soudard ${ }^{13}$; le propos semble ici plus offensif, mais ne fait qu'exprimer incidemment une raillerie traditionnelle. L'appartenance de ces personnages au monde judiciaire relève donc pour une bonne part de l'accident et manifeste d'autant moins une posture critique que l'action se déroule en dehors de toute scène juridique : la rue (XI), l'appartement privé (XXV), le jeu de paume et une arrière-boutique $(\mathrm{X})$, un lieu indéterminé (LXVI).

Cette catégorie du satirique a été sur divers registres privilégiée par les lecteurs modernes ${ }^{14}$. Il importe cependant de ne pas banaliser le propos de Des Périers : traiter uniformément les récits qui ont trait à l'univers juridique occulterait l'interprétation plus radicale à laquelle donnent prise ceux qui se situent au cœur des problématiques du droit et de la justice.

On opposera par exemple aux précédentes l'aventure de Maistre Arnaud. Celui qu'on nous présente comme un «legiste» interprète à son profit l'un des adages les plus courants, Qui tacet consentire videtur (XXIV, p. 116); or cet adepte des «bons tours » (p. 114) justifie par cet instrument l' «emprunt» d'une haquenée durant neuf mois puis abuse sa victime au point d'obtenir d'elle des remerciements ; la fin du conte nous apprend que cette tromperie n'est que la première d'une longue série. Explicitement rapprochée des «tours villoniques» de maistre Pierre Faifeu (XXIII, p. 109), dont l'histoire précède, cette malhonnêteté, loin de recevoir quelque sanction que ce soit, est source de grande réjouissance ${ }^{15}$. On confrontera ces deux contes picaresques à la nouvelle sinistre où, pour un motif identique, se déchaîne une violence judiciaire réprouvée par le conteur-devisant : comme ces deux farceurs, c'est précisément une jument qu'a volée Jean Trubert, récidiviste comme eux, et ce n'est pas à un «povre homme» (p. 109) mais à un «riche paysant», déclenchant

\footnotetext{
${ }^{11}$ Cf l'analyse de $\mathrm{Ph}$. de Lajarte, «Propositions pour la construction d'un modèle d'analyse du conte facétieux en tant que genre ", Facétie et littérature facétieuse à l'époque de la Renaissance. Actes du colloque de Goutelas, 1977, RHR n ${ }^{\circ}$ 7, 1978, p. 122-129.

${ }^{12}$ Cette observation vaut aussi pour le clerc du procureur au Châtelet qui se venge de mauvais traitements en faisant croire à son maître qu'il est sourd et qui sera durement châtié de cette «chatterie» (57), ainsi que pour le juge d'Aigues Mortes et son collègue non identifié (LXVI ; v. Sozzi, Les Contes..., op. cit., p. 139-140).

${ }^{13}$ LXXIII, 264. Il faut rapprocher ce passage des dictons de la nouvelle LXVII, p. 249 et 250 , ainsi probablement que de l' « affection » exagérée des plaidoyers (XVII, p. 90).

${ }^{14}$ Sur cette veine, voir L. Sozzi, Les Contes de Bonaventure Des Périers, op. cit., p. 286 et p. 377-378, et K. Kasprzyk, éd. cit., p. XXXVII-XXXVIII.

${ }^{15} \mathrm{P}$. Mounier donne une analyse plus subtile de la nouvelle : «Densité narrative et dissonance énonciative : le début de l'histoire de maître Arnaud. Étude stylistique d'un extrait de la nouvelle 24 », Lire les Nouvelles Récréations et Joyeux Devis de feu Bonaventure des Périers, sous la dir. de B. Boudou et D. Bertrand, Presses de l'Université Blaise Pascal, Clermont-Ferrand, 2009, p. 215-240.
} 
ainsi de la part de la justice la répression dont son fils, le "povre petit» (LXI, p. 227, p. 229), sera la victime collatérale.

Il faut se garder d'inférer de ces inégalités criantes une prise de position explicite de l'auteur dans une œuvre à dominante facétieuse et délibérément composite $^{16}$. On peut cependant soupçonner que Des Périers, qui relève à deux reprises le privilège du for (XXVIII, p. 132-133 et LXXXI, p. 289) et cite comme un dicton l'inégalité de l'exercice de la justice ${ }^{17}$, est sensible à cette réalité, même dans un contexte d'hétérogénéité du système judiciaire ${ }^{18}$. À la faveur des rencontres auxquelles invite la forme du recueil ${ }^{19}$ apparaît l'extrême variabilité d'un droit qui se prête, ces cas le montrent, à l'abus privé, au juste châtiment d'un malfaiteur, à la brutalité inique envers un innocent aussi bien qu'à l'impunité. Si chacune de ces nouvelles ne parle que pour elle-même, leur confrontation par un lecteur invité à participer à la production du recueil peut s'avérer confondante.

La vertu révélatrice de ces épisodes est particulièrement forte lorsque l'action se joue, comme dans le cas de Jean Trubert, sur la scène judiciaire ou dans sa proximité. À un accusé qui doit comparaître pour un baiser volé, l'on conseille de s'éloigner car son accusatrice est «apparentée des Juges et des Advocatz» (LXXVIII, p. 278). Que ce soit un lieu dangereux est confirmé par la série des voleurs: un prisonnier qui fait des révélations «soubz ombre qu'on luy promit impunité» est pendu, principalement pour avoir volé un curé qui trafiquait «les biens de l'eglise» (LXXIX, p. 280 et 282); un autre auquel « on promit, selon la coustume, qu'il n'auroit point de mal, s'il vouloit parler rondement » subit le même sort après quelques «jeux» du prévôt (LXXXII, p. 289); le prévôt La Voulte se venge «par un beau depit» du vol dont il est victime en multipliant les pendaisons sans jugement (LXXX, p. 285); enfin, un bandoulier, «hardy Capitaine... cassé avec d'aultres, quand les guerres furent finies » et «sommairement» jugé, voit sa peine aggravée après le prononcé de la sentence, pour un bon mot qu'il commet (LXXXII, p. 291). On constate ailleurs comment Coquillaire et Maillart, dont est soulignée la pratique expéditive, usent de tromperie pour convaincre un coupable (XXVIII, p. 132 et 133), et la sottise d'un bâtard prêt à se laisser exécuter par orgueil révèle les manières expéditives d'un «pendeur de gens » (XLIV, p. 183). Loin d'être réductible à la satire des gens de justice, l'accumulation de ces cas dévoile le visage d'un appareil judiciaire partial, fallacieux dans ses procédés $^{20}$, sourd aux

\footnotetext{
${ }^{16}$ Ces nouvelles appartiennent à des courants radicalement différents, comme l'annonçait le prologue : B. Des Périers, Nouvelles Récréations et Joyeux Devis, I, p. 15 : « Il y en ha de tous boys :... fors que pour plorer. ».

17 Ibid., LXXXII, p. 290 : «car on dit, rigueur de Thoulouse, humanité de Bordeaux, misericorde de Rouan, justice de Paris... ».

${ }^{18}$ L'éditrice et L. Sozzi notent souvent, à juste titre, que les parlements bénéficient d'un meilleur traitement que la justice prévôtale. Les exemples que nous analysons le confirment tous.

${ }^{19}$ Et que le conteur souligne ainsi : «Ouvrez le livre : si ung compte ne vous plait, hay à l'aultre. », I, p. 15.

${ }^{20}$ V. N. Lombart, pour qui « L'action des coupeurs de bourses aboutit à la révélation, au sens fort du terme, du visage agressif et sournois de la justice.», «L'éloge de l'habileté
} 
circonstances atténuantes suggérées par le conteur et finalement plus préoccupé de la perte du «justiciable» que de l'établissement de la justice: elle révèle une perversion profonde de la loi.

La question de l'ethos et du caractère même des juges n'est pas indifférente ${ }^{21}$. Ceux-ci apparaissent souvent corrompus par la passion, comme le président de la nouvelle XC, implicitement classé parmi les «mauvais folz et dangereux » (XC, p. 310) ${ }^{22}$, comme La Voulte, "un mauvais et dangereux fol, avec son col tors » (LXXX, p. 284). Beaucoup laissent leurs affects influencer leur jugement : on vient de le voir, les peines sont souvent motivées par la colère ou le caprice des juges. Bien loin de connaître la joie de Socrate et du Plaisantin (p. 17-18), ceux-ci sont toujours agélastes, ce qui invite à les rapprocher des « frons rechignez » (p. 2), de la « tétricité» (p. 18) que le recueil veut conjurer.

Ces récits confirment également ce que révélait le cas de maistre Arnaud : la corruption du langage. Une pesante métaphore l'illustre chez le procureur en quête d'une « chambrière » : l'intrigue s'amorce sur son habitude de revisiter « les proces de quelques femmes veufves, et aultres qui venoient chez luy pour le solliciter», mais « il luy coustoit à fournir à l'appointement»; la transaction avec la mère de sa victime, «contraincte par povreté », s'opère dans un langage dissimulé (VIII, p. 48, p.49) et la métaphore reparaît pour narrer comment le clerc a commencé à «deniaisier» l'innocente: il «prenoit ceste charge sans procuration» (p. 51) ${ }^{23}$. C'est, sur le mode comique, la mise en cause de la valeur de vérité du langage juridique: il n'engage pas la foi de celui qui l'utilise comme l'ont montré les mensonges des juges et, de l'étudiant légiste qui apprend à «lire» devant des choux (LXXVI) à l'avocat qui parle à sa chambrière en latin (XIV), il est sujet à de constants déplacements, des «métaphores » qui n'ont rien de poétique puisqu'elles visent exclusivement à la satisfaction d'intérêts personnels par le transfert illégitime du langage sur des cas singuliers auxquels est intéressé celui qui en use. Le jeu de mots de Jacques Colin, «homme de bon sçavoir et de bon cerveau », confondant

mercurienne dans les Nouvelles Récréations et joyeux devis : Bonaventure Des Périers face aux «coupeurs de bourses» (nouvelles LXXIX, LXXX, LXXXI)», Lire les Nouvelles Récréations et Joyeux Devis..., op. cit., p. 103.

${ }^{21}$ On loue ailleurs la clairvoyance de Coquillaire qui « voyoit assez clair dedens un sac des yeulx de l'esprit » (XXVIII, p. 133), mais le mal qui affecte ses yeux vient la contredire en révélant sa crédulité : il n'est pas «fin», et s'il est «bon justicier», il faut sans doute entendre qu'il s'agit de son habileté procédurière. Le récit induit ainsi le lecteur attentif à supposer son absence de jugement et s'achève par une phrase ambiguë dont on ne sait si elle loue ou fustige la manière dont le juge traite les accusés.

${ }^{22}$ La distinction essentielle entre les deux sortes de folie a été établie par R. Aulotte, «Fous et farceurs dans les Nouvelles Récréations de Bonaventure des Périers », Le Génie de la forme, Mélanges de langue et littérature offerts à Jean Mourot, Presses Universitaires de Nancy, 1982, p. 97-106.

${ }^{23}$ Le même dévoiement apparaît chez l'avocat stagiaire qui imite la langue juridique pour séduire la femme d'autrui : "Les trois pointz que vous m'alleguez, ma dame...» (XVI, 86); v. V. Montagne, «La rhétorique de la séduction dans la nouvelle 16 des Nouvelles Récréations et joyeux devis de Bonaventure Des Périers ", Lire les Nouvelles récréations et joyeux devis..., op. cit., p. 197-213. 
« débouter » et «débotter » (XLVII, p. 192, p. 194) est-il dès lors si inoffensif qu'il paraît $^{24}$ ? Si l'humaniste, mettant en pratique les recommandations du prologue, prend l'affaire sur le mode du rire, cette justice apparaît comme une justice qui « débotte », c'est-à-dire qui dépouille son patient, exactement comme maistre Pierre Faifeu sa victime (XXIII, p. 111) : affaire de «tours », et non plus de justice.

Si ce n'est leur exclusivité, les gens de loi, qui figurent, du point de vue de la satire, au premier rang des ignorants vaniteux, sont aussi les premiers à jouer avec les propriétés du langage pour faire de celui-ci un usage incongru ou immoral ${ }^{25}$ et c'est sur eux que se concentrent les traits de la cruauté et l'injustice ${ }^{26}$.

La condamnation de l'institution judiciaire n'est cependant pas univoque car plusieurs témoignages plaident en sa faveur: un conseiller fait preuve de bienveillance envers une pauvre vieille ignorante (XLII, p. 179); face au «fol adjournement » de Chichouan qui assigne son beau-père parce qu'il tarde à mourir, le juge sait «moderer» sa sentence (XLIX, p. 201); la cour statue avec la même sagesse sur une affaire de baiser (LXXVIII, p. 279); le parlement de Paris est incidemment loué pour sa pratique scrupuleuse (XVII, p. 90) ${ }^{27}$.

Une figure éminente se distingue : c'est le «personnage de robbe longue et de Justice» (VI, p. 39) qui corrige les infidélités de son épouse ${ }^{28}$. Réunissant une cour familiale, il la place face à ses responsabilités et la contraint doucement au repentir et au rachat, exerçant ainsi une justice efficace et mesurée. Cet «homme de bon discours » a pratiqué une ruse qui n'a pas pour but de convaincre ni de châtier la coupable, mais de l'amener à s'amender elle-même. Récompensé par un roi luimême exemplaire, il incarne probablement aux yeux de Bonaventure des Périers

\footnotetext{
${ }^{24}$ Celui qui précède, sur les moines/demoniorum, l'est-il d'ailleurs ? Dans ces deux cas, Jacques Colin que l'on ne peut soupçonner d'ignorer le latin, joue de cette langue pour émettre deux jugements sévères. Ce latin génère d'ailleurs une réflexion sur la langue de la justice, sur laquelle nous reviendrons (XLVII, p. 194).

${ }^{25} \mathrm{~K}$. Kasprzyk met à juste titre l'accent sur le fait que les ratés du langage révèlent une inaptitude fondamentale à la communication attachée à la condition humaine (« Des Périers et la communication ", Études seiziémistes offertes à V. L. Saulnier, Genève, Droz, 1980, p. 169-178), mais il faut clairement distinguer ce qui relève de l'incommunicabilité et les récits qui, comme ceux-ci, montrent au contraire un singulier talent à mal user du langage.

${ }^{26}$ K. Kasprzyk, éd. cit., p. XXXVIII, et L. Sozzi, op. cit., p. 377.

${ }^{27}$ Certaines notations indicielles sont plus délicates à interpréter : par exemple, faut-il donner un sens au fait que la sage dame de la nouvelle XXXVIII est une «baillive »? À l'inverse, faut-il s'arrêter sur le fait que celui qui apprend la médecine par appât du gain est un écolier légiste (LIX) ?

${ }^{28}$ Nous renvoyons à la lecture, sensiblement différente, qu'en donne D. Ménager, «La sixième nouvelle des Joyeux Devis », Lire les Nouvelles récréations et joyeux devis..., op. cit., p. 183-194 ; v. aussi G. Demerson, «Bonaventure Des Périers : la nouvelle, art de la patience », Proses et prosateurs de la Renaissance. Mélanges Robert Aulotte, Paris, SEDES, 1988, p. 68, et B. Boudou, Bonaventure des Périers. Nouvelles Récréations et Joyeux Devis, Neuilly, Atlande, 2008, p. 200-201.
} 
l'idéal du justicier ${ }^{29}$. Son opposé exact est le magistrat de la dernière nouvelle ${ }^{30}$, qui exerce sur sa femme un cruel châtiment. Ces deux présidents présentent deux visages opposés de la justice: une justice publique, réparatrice ${ }^{31}$ fondée sur des normes morales ${ }^{32}$ et manifestée par un discours de sagesse ${ }^{33}$; et une justice secrète ${ }^{34}$ et muette, cruelle, irréversible ${ }^{35}$ et purement vindicative.

Il se trouve que chacun de ces contes, aux deux extrémités du recueil, affecte, dans le registre de la gravité, une forme qui déroge au genre des Nouvelles Récréations et Joyeux Devis : l'histoire tragique (XC), en passe de s'affirmer comme le grand genre de l'exemplarité et du châtiment, et le récit encadré (VI), le seul du recueil ; la mise en scène du narrateur second, un homme sérieux, occulte dans cette dernière la présence du conteur facétieux et l'encadrement désigne sans équivoque la nouvelle, genre dont l'obsolescence souligne l'irréalité d'un « jadis » insituable ${ }^{36}$.

\footnotetext{
${ }^{29}$ On notera que l'identité du personnage est indifférente dans les analogues généralement invoqués ; l'encadrement du récit par le thème judiciaire (p. 39 et p. 43) est ajouté par Des Périers. D’une manière plus générale, la documentation réunie sur les «sources » et sa discussion (dans les travaux de J. W. Hassel et L. Sozzi) montrent que la distribution des acteurs est de son fait ; cette inflexion notable retentit sur l'appréciation portée sur le monde judiciaire. Toutefois, pour la nouvelle CX, le personnage est dès la source un «president» ( $47^{\mathrm{e}}$ des Cent Nouvelles nouvelles).

${ }^{30}$ Pour son interprétation, voir B. Boudou, «Patience ou passion dans la dernière des Nouvelles Récréations et Joyeux Devis de Bonaventure des Périers ", RHR $\mathrm{n}^{\circ}$ 67, décembre 2008, p. 123-138, et Bonaventure des Périers..., op. cit., p. 95-96 et p. 201-202.

${ }^{31}$ La ruse du personnage a un but pédagogique, ses réquisitions sont a minima (p. 42), et tout cela conduit à un amendement de la coupable par elle-même (p. 42-43).

${ }^{32}$ Il faut voir combien cet homme en est soucieux : face à l'infidélité, il s'accuse lui-même d'avoir commis la « follie » de choisir une épouse trop jeune (il n'a pas enfreint une loi, mais une norme alors dictée par les convenances et le «bon sens ») ; lorsqu'un parent lui en parle, il «fait semblant devant» lui «tel que le cas le requeroit»; il pense que ce ne sont pas les interdits qui assurent la fidélité d'une femme, mais les « respect de la vertu, et... crainte de son deshonneur », etc. (p. 40-41).

${ }^{33}$ On notera qu'au contraire de beaucoup de personnages peu recommandables, il n'use pas du langage juridique ; s'il recourt à un énoncé normatif, c'est au proverbe, qui manifeste la sagesse des nations : «Si est-ce qu'il vaut mieux tard que jamais » (p. 42). On pourra lui opposer le séduisant avocat Beaufort.

${ }^{34} \mathrm{Cf}$. la nouvelle où le prévôt fait dépendre sa victime « de peur qu'il en fust nouvelles. » (LXI, p. 229)

${ }^{35}$ Comme pour le fils de Jean Trubert : «.... seroit une belle deffaicte que d'un jeune loup », dit le prévôt (comprendre : un bon débarras); et le conteur d'ajouter : "Voyla toute la recompense qu'eut le povre petit, excepté que le Prevost le fit despendre : de peur qu'il en fust nouvelles. » (LXI, p. 229) : pas de réparation, donc, pour la victime de l'erreur judiciaire. Nous avançons ici une lecture différente de celle que proposent A. Blanckaert et R. Weber : «Nouvelles Récréations et joyeux devis: pour qui ? pourquoi?», Lire les Nouvelles récréations et joyeux devis..., op. cit., p. 58.

${ }^{36} \mathrm{Ce} «$ jadis » (p. 38) exclut François Ier. Il faut une petite enquête pour découvrir qu'il s'agit probablement de Louis VI le Gros, selon le témoignage de Jean Bouchet (Les anciennes et modernes gesnealogies des Roys de France et mesment du Roy Pharamond, avec leurs
} 
Il se trouve en outre que la quasi totalité des cas rapportés dans le recueil, entre ces deux modèles extrêmes de la justice, fait pencher la balance du mauvais côté, comme pour une condamnation définitive : la nouvelle dessinait nostalgiquement les traits d'une justice idéale, l'histoire tragique en peint la triste réalité, comme en un résumé sinistre des cas égrenés dans les contes.

Les perversions de l'institution et de ses acteurs démentent l'idée même de justice, qui déserte la scène judiciaire; quand l'on y voit en permanence s'exercer l'iniquité, c'est ailleurs et sous d'autres formes que la justice sera mise en œuvre. Celle, parfaite, qu'exerce le président exemplaire en inventant une cour familiale aristocratique et impartiale (VI, p. 41) est exceptionnelle. Partout ailleurs, la délocalisation de la justice signe le retour d'une forme primitive, faite d'intimidation $^{37}$, de rétorsion ${ }^{38}$, de châtiment corporel directement infligé au coupable par sa victime ${ }^{39}$. Cette régression vers la vengeance réduit la force publique du droit à la violence privée, ce dont le mauvais président de la nouvelle finale est encore une illustration : habité par une passion vindicative longuement entretenue, il met à profit une «vaccation de Court» $(\mathrm{XC}, \mathrm{p} .311)$ pour frapper, à l'écart de tout. La justice, injuste sur la scène judiciaire, se résout ainsi, lorsqu'elle s'exerce en dehors d'elle, dans l'injustice d'un monde d'arbitraire et de violence. Au mieux l'on goûtera les bons tours des humbles ${ }^{40}$ qui, dans la sujétion à de plus puissants, n'ont d'autre recours que d'astucieuses vengeances.

L'impuissance de l'institution judiciaire est à la mesure de son injustice. C'est l'un des sens possibles, entre cour de parlement et dîner en ville, de l'affaire des barbes: l'édit les concernant est tombé en désuétude et la loi a cédé devant l'usage arbitraire qu'est «la mode de court» (XVII, p. 91). Quant aux prévôts, si prompts à pendre des innocents, saura-t-on jamais s'ils arrêtent les brigands : «...les trouva il ? Et qu'en sçay je mon ami? je n'y estois pas. » (XX, p. 101) ? Une autre marque d'inefficience est l'incompréhension que suscite la loi : elle ne savait [SAIT] se faire entendre des justiciables, mais la décision louable de mettre « les arrestz en bon Françoys » (XLVII, p. 194) ne semble pas y avoir remédié, comme en témoignent diverses situations d'incompréhension ${ }^{41}$. Son incapacité à se faire

epitaphes et effigies, Poictiers, Jacques Bouchet, 1527, fol. 106 ${ }^{\vee}$ ), confirmé, dans une interprétation moins positive, par Gilles Corrozet (Les divers propos mémorables des nobles et illustres hommes de la chrestienté, Paris, Gilles Corrozet, 1557, p. 32).

${ }^{37}$ Comme, sur le mode comique, celle qu'un seigneur veut exercer à l'endroit des pages qui ont cloué l'oreille d'un fou à un poteau ; la justice repose, et d'ailleurs en vain, sur la crainte du fouet plutôt que sur la force de la loi et de la morale (II, p. 19-20).

${ }^{38}$ "...les gaudisseries retournent quelque foys sus leurs gaudisseurs » (XXVI, p. 125).

39 Par exemple, un gentilhomme essorille sur le champ un coupeur de bourse (LVI) ; B. Boudou y voit l'illustration d'un abus arbitraire (Bonaventure des Périers..., op. cit., p. 132-33).

${ }^{40}$ Entre autres Gillet le menuisier et le savetier Blondeau (XVIII et XIX), mais le schéma est fréquent et apparaît dès la nouvelle III.

${ }^{41}$ Une vieille cherche un conseiller « laid» (XLII), occasion pour noter les méandres de la justice ; le beau-père de Chichouan ne comprend pas pourquoi il est «ajourné » (XLIX, p. 200) ; un Poitevin exécuté par un sergent s'attend à voir sa charrette et ses bœufs « en la 
comprendre vient donc compléter sa capacité à générer des abus. Cette justice est par ailleurs dépourvue d'exemplarité : redoutée des honnêtes gens ${ }^{42}$, elle n'intimide ni le curé de Brou défiant son évêque qui le menace de prison (XXXIV, p. 155), ni aucun des coupeurs de bourse que nous avons cités, la nouvelle LXXX montrant même, à l'étonnement admiratif du conteur, ces hommes de l'art accourant au procès d'un de leurs confrères en quête d'une «bonne fortune» : «...qui penseroit qu'il y en eust de si hardis de desrober au propre lieu où se fait le proces d'un larron?» (p. 284) ? $^{43}$. Mais sa plus grande défaite est sans doute l'impunité. Celle-ci est d'un tout autre ordre que celle que l'on peut rencontrer dans le genre tragique (histoires tragiques, canards criminels) : ce scandale appellera un châtiment suprême au nom du principe que «Dieu punit tout quand il lui plaît», les délais de la justice divine palliant en quelque sorte les failles de celle des hommes. Ici, aucune perspective eschatologique n'offre d'échappatoire: l'impuni le restera et il n'est d'autre issue que d'en rire, en faisant au passage bon marché de la justice. On pourra en voir une illustration - en s'interrogeant, ici comme dans bien d'autres cas, sur la nature comique de la nouvelle - dans le cas de l'honnête tailleur auquel deux écoliers «bons frippons » volent sans qu'il y puisse rien faire son outil de travail (LXXXIV, p. 293).

Lorsque le conteur, dans un geste singulier (« quant à moy»), pour défendre sa liberté d'invention, se met à l'écart des « faiseurs de contractz et... intenteurs de proces » (p. 15), il livre une clé de lecture importante. Des lois régissent la vie des hommes, et cette prise de distance n'en conteste pas la nécessité, se bornant à ironiser sur les chicaneurs, dont un évêque aux mille procès illustrera les extrémités (XXXIV, p. 157-158). Ce ne sont pas les principes du droit qui sont en cause mais, comme on l'a vu, les modalités de l'exercice de la justice, qui la dénaturent profondément.

Pour les lois qui régissent le discours, il en va autrement; plusieurs d'entre elles sont délibérément rejetées dans le prologue : la nécessité de l'ordre et le critère de vérité (p. 15), la validité des autorités, telles que Platon et Xénophon (p. 17-18), enfin le principe de non contradiction; et il l'est précisément sur ce point de la vérité : d'une page à l'autre son importance se trouve niée ${ }^{44}$ puis implicitement

main du roi » (LXX, p. 258); un avocat parle à sa chambrière en latin et doit utiliser son clerc comme truchement (XIV) et, plus tragiquement, le fils de Jean Trubert est pendu parce que prévôt et greffier ne se sont pas entendus sur le sens d'une préposition (LXI, p. 229).

${ }^{42}$ Comme dans l'affaire du baiser, LXXVIII, p. 278.

43 J.-P. Siméon voit dans ce détail un témoignage sur l'audace des classes dangereuses («Classes sociales et antagonismes sociaux dans les Nouvelles Récréations et Joyeux Devis de Bonaventure des Périers », La Nouvelle française à la Renaissance, éd. L. Sozzi, Genève, Slatkine, 1981, p. 330). À bien la lire, cette nouvelle propose une exemplarité inversée : la foule veut connaitre cet «homme de mestier » qu'est le coupeur de bourse et voir « la façon $\mathrm{du}$ Prevost, qui estoit mauvais et dangereux fol, avec son col tors» (p. 284) ; c'est la valorisation mercurienne du voleur dont parle N. Lombart, art. cit., p. 99 sq.

${ }^{44}$ B. Des Périers, Nouvelles Récréations et Joyeux Devis, I, p. 15 : «Qu'on ne me vienne non plus faire des difficultez: Oh ce ne fut pas cestuy cy qui fit cela... [...] Riez seulement, et ne 
soulignée par l'image des «pierreries falsifiées» (p. 16), après avoir été altérée par un tour de passe-passe qui lui donne comme seule mesure le plaisir du lecteur ${ }^{45}$. Les « feintes » du narrateur ${ }^{46}$ instaurent une forme singulière de vérité en substituant aux règles du discours commun celles de la fiction, qui autorise l'invraisemblance ${ }^{47}$ et offre la liberté de jouer de l'invention et de l'approximation ${ }^{48}$. La falsification, réprouvée dans le monde ${ }^{49}$, est licite dans le discours du conteur-devisant; son statut l'affranchit d'une loi de vérité que l'institution judiciaire aurait, dans le monde réel, le devoir d'observer pour elle-même et de défendre pour la société, quand tout montre qu'elle n'accomplit pas cette mission.

Cette distinction entre l'espace de la fiction et la réalité met en cause les formes de la loi et sa nature. Dans l'espace de la fiction se manifestent une loi et un jugement souverains, portés par la parole volubile d'un conteur-devisant qui joue avec son public. Loin des formes sclérosées d'une langue juridique dont les récits montrent l'instrumentalisation et le dévoiement, sa morale universelle, invariable et simpliste, formulée dans le prologue puis mise en pratique dans le discours, s'impose de sa propre autorité : c'est le «Bene vivere et letari» qui s'oppose aux préceptes aussi discordants qu'impératifs des philosophes («il fault», p. 14); c'est «le plus gentil enseignement pour la vie» décrète le conteur, et sa validité s'affirme dans toute sa grâce face aux divers «notables ».

Plusieurs contes mettent en œuvre des jugements extrajudiciaires qui manifestent la supériorité de cette loi universelle sur l'appareil juridique : le père des filles débauchées refuse de se glisser dans la peau d'un juge, comme les «peres rigoureux », et préfère "prendre peine... pour raccoustrer les choses» (V, p. 35), exerçant dans sa grande sagesse une forme de justice essentiellement réparatrice, fondée sur la loi naturelle. Le premier acteur qui se manifeste dans le recueil, le

vous chaille si ce fut Gaultier ou si ce fut Garguille. » [...] «Quant à moy, je ne suis point si scrupuleux. »

${ }^{45}$ Ibid., p. 16 : « ...peult estre qu'il n'est pas vray. Que me chault il, pourveu qu'il soit vray que vous y prenez plaisir? » Voir aussi, adressé aux demoiselles, p. 17 : « Lisez hardiment... [...] Lisez tout, lisez lisez... [...] Ne les lisez donc pas ».

${ }^{46}$ Voir l'analyse de M. Jeay, «Les feintes du narrateur dans les Nouvelles Récréations et Joyeux Devis et le Cymbalum mundi », Méthode! n 14, Vallongues, 2009, p. 81-93.

${ }^{47}$ B. Des Périers, Nouvelles Récréations et Joyeux Devis, I, p. 16 : «...j’ay voulu faindre quelques noms tout expres pour vous monstrer qu'il ne faut point plorer de tout cecy que je vous compte : Car peult estre qu'il n'est pas vray. »

${ }^{48}$ Par exemple, ibid., XXVII, p. 131 : «Les uns me conseilloyent que je disse que cecy estoit advenu en yver pour mieulx faire valoir le compte. Mais estant bien informé que ce fut en esté, je n'ay point voulu mentir, Car avec ce qu'un compte froid n'est pas trouvé si bon : Je me damnerois : ou pour le moins il m'en faudroit faire penitence. Toutesfois il sera permis à ceulx qui le feront apres moy de dire que ce fut en yver, pour enrichir la matiere. Je m'en rapporte à vous. » Le souci de vérité est tourné en dérision par une discussion sur un détail indifférent, ibid., XXXVII, p. 163-164: «Aulcuns ont voulu dire que la mule n'estoit pas grise : et qu'elle estoit noire. Mais il y ha des gens qui ont un esprit de contradiction dedans le corps. Et qui voudroit contester avec eulx, ce ne seroit jamais faict. »

${ }^{49}$ Idée illustrée par les deux nouvelles en forme de fable qui mettent en jeu les alchimistes, XII et XIII. 
Plaisantin, incarne celle-ci à la perfection : il «raille» au moment de l'extrême onction, et oppose à la réprobation du prêtre une «felicité » inaltérable; il bouscule toutes les normes humaines, jusqu'au cérémonial de la bonne mort et son sens spirituel, au nom de sa soumission à la loi naturelle ${ }^{50}$. Comme il «mourut plaisamment » (p. 18), il réfute à sa manière le principe de non contradiction et de nombreux épisodes témoigneront ainsi de la prévalence des valeurs naturelles sur l'artifice des normes ${ }^{51}$.

Quand tant de cas témoignent de la défaillance de l'institution judiciaire, la loi qui apparaît véritablement juste et légitime, abstraite des formes juridiques, relève donc des principes naturels soutenus par le conteur ${ }^{52}$, conformément à des critères éthiques dont la pureté est soulignée par la simplicité de leur énoncé ${ }^{53}$. Audelà du jeu sur les normes qu'offre la liberté facétieuse, les Nouvelles récréations et joyeux devis montrent par une représentation dénuée de didactisme, dans l'espace de liberté à part soi que dégage la fiction, une forme de vérité et de justice supérieures. L'absence délibérée de conformité du recueil à la vérité commune lui ouvre la possibilité de manifester par des contes à rire le défaut manifeste de justice du monde réel et d'affirmer sans enseigner l'existence de la Justice. Face à une justice humaine profondément viciée, la falsification fictionnelle apparaît comme la source d'une vérité que formulent les contes, une vérité plus vraie que la fausse vérité de la justice ${ }^{54}$.

La force de la facétie s'exerce donc par le renversement des valeurs de vérité, qui ne sont plus détenues par la justice et son grave discours, mais par la parole légère du conteur, ou simpliste et naturelle de ses personnages. Une fable dit le caractère extrêmement douteux de la justice : la nouvelle XXIX - qui pour sa complexité mériterait à elle seule une étude - montre le personnage parfaitement ambigu d'un renard qui appartient à un bailli et dont sont retracés les exploits ou les méfaits puis le procès et l'exécution controversés. Sa clôture caricaturalement

\footnotetext{
${ }^{50}$ «Chose qui luy estoit si naturelle et si propre... » et «Que voulez vous de plus naif que cela ? » sont les deux phrases qui encadrent la fable (p. 18-19).

${ }^{51}$ D'autres scènes de jugement extrajudiciaire vont dans le même sens, comme le décret du cardinal de Luxembourg qui cède son prénom au fils d'une «bonne femme» (XV), le jugement prononcé par le savetier Blondeau qui noie le trésor dont la découverte l'a rendu mélancolique (XIX, p. 97), le procès de la danse (XXXVIII), le verdict plein de bon sens de l'abbesse face à l'intrusion d'un jeune homme (LXII, p. 232). Ces récits confirment, en dehors du cadre juridique, la force de la loi naturelle qu'indiquait le bon président de la nouvelle VI.

${ }^{52}$ Sur cette valorisation de la loi naturelle, v. L. Sozzi, op. cit., p. 395-399 et N. Le Cadet, «L'éloge de la naïveté dans les Nouvelles récréations et joyeux devis », Lire les Nouvelles récréations et joyeux..., op. cit., p. 71-90.

${ }^{53}$ B. Des Périers, Nouvelles Récréations et Joyeux Devis, I, p. 15 : «Je vous promets que je n'y songe mal ni malice »; ibid., p. 17 : « il n'y ha rien qui ne soit honneste».

${ }^{54} \mathrm{~V}$. la distinction de D. Ventura entre vérité factuelle et vérité du monde (Fiction et vérité chez les conteurs de la Renaissance en France, Italie, Espagne, Presses Universitaires de Lyon, 2002, p. 108).
} 
exemplaire (« Voilà comment n’y ha finesse ne mechanceté qui ne soit punie en fin de compte », p. 139) souligne l'ironie qui la parcourt tout entière.

La critique radicale de la loi et de la justice rend en quelque sorte justice : à la justice et à la vérité, mais aussi aux victimes d'une justice injuste, qui trouvent ici, dans l'univers de la fiction, comme une compensation des torts qu'elles ont subis. On conviendra, avec Des Périers, que «c'est un pauvre reconfort à un homme, que sa mort sera bien vengée » (XLIV, p. 185). Ce sont là les limites de la puissance du conteur facétieux, qui n'est précisément pas un justicier ${ }^{55}$. Au moins cela peut-il être retenu à la fois comme une prise de position de principe, une réaction dysphorique face à un état de faits insupportable ${ }^{56}$, et une forme de réparation morale pour les victimes de la justice: avec le rire, avec la parole facétieuse, les principes de la justice auront eu le dernier mot et la facétie aura vengé, dans la fiction, les victimes de la réalité.

L'expérience de la justice imaginée et transcrite dans les Nouvelles récréations et joyeux devis conduit apparemment à une conclusion désespérée, dont il faut s'empresser de rire: de justice, il n'y a point, et surtout pas chez les hommes et les institutions qui ont pour mission de la faire régner. Au fil des «essais» auxquels elle soumet la justice, la rencontre des hommes de loi et des hors-la-loi dévoile ainsi l'imposture de l'institution ${ }^{57}$ et révèle l'inanité de la justice forgée par les hommes dans les formes du droit, auxquelles le conteur-devisant oppose un rire impuissant mais réconfortant et l'affirmation de valeurs naturelles universelles.

Jean-Claude Arnould Université de Rouen

ANR Juslittera

\footnotetext{
${ }^{55}$ Notre lecture va à l'encontre de celle de J.-P. Siméon, qui lit le châtiment des coupables comme une contribution du conteur à la défense de la collectivité et voit donc en Des Périers un défenseur de l'ordre établi (art. cit., p. 345 et p. 350-351).

${ }^{56}$ On pense à l'attitude rabelaisienne décrite par G. Demerson, «Rabelais et la violence », Humanisme et facétie, Quinze études sur Rabelais, Orléans, Paradigme, 1994, p. 291-307.

${ }^{57}$ On peut y voir un trait de cynisme. La parenté est forte avec la dénonciation cynique du charlatanisme, religieux par exemple (M. Clément, Le Cynisme à la Renaissance, Genève, Droz, 2005, p. 111 sq.) et la forme de la leçon de Des Périers a beaucoup à voir avec celle de l'enseignement cynique (ibid., p. 62-63). Sur le cynisme propre à la facétie, v. G. Demerson, «Les facéties chez Rabelais », op. cit., p. 53.
} 